\title{
OPEN Prognostic and therapeutic implications of extracellular matrix associated gene signature in renal clear cell carcinoma
}

\author{
Pankaj Ahluwalia ${ }^{1}$, Meenakshi Ahluwalia ${ }^{1}$, Ashis K. Mondal ${ }^{1}$, Nikhil Sahajpal ${ }^{1}$, Vamsi Kota $^{2}$, \\ Mumtaz V. Rojiani ${ }^{1}$, Amyn M. Rojiani ${ }^{1}$ \& Ravindra Kolhe ${ }^{1 \bowtie}$
}

Complex interactions in tumor microenvironment between ECM (extra-cellular matrix) and cancer cell plays a central role in the generation of tumor supportive microenvironment. In this study, the expression of ECM-related genes was explored for prognostic and immunological implication in clear cell renal clear cell carcinoma (ccRCC). Out of 964 ECM genes, higher expression (z-score > 2) of 35 genes showed significant association with overall survival (OS), progression-free survival (PFS) and disease-specific survival (DSS). On comparison to normal tissue, 12 genes (NUDT1, SIGLEC1, LRP1, LOXL2, SERPINE1, PLOD3, ZP3, RARRES2, TGM2, COL3A1, ANXA4, and POSTN) showed elevated expression in kidney tumor $(n=523)$ compared to normal $(n=100)$. Further, Cox proportional hazard model was utilized to develop 12 genes ECM signature that showed significant association with overall survival in TCGA dataset ( $\mathrm{HR}=2.45 ; 95 \% \mathrm{CI}[1.78-3.38] ; p<0.01)$. This gene signature was further validated in 3 independent datasets from GEO database. Kaplan-Meier log-rank test significantly associated patients with elevated expression of this gene signature with a higher risk of mortality. Further, differential gene expression analysis using DESeq2 and principal component analysis (PCA) identified genes with the highest fold change forming distinct clusters between ECM-rich high-risk and ECM-poor low-risk patients. Geneset enrichment analysis (GSEA) identified significant perturbations in homeostatic kidney functions in the high-risk group. Further, higher infiltration of immunosuppressive T-reg and M2 macrophages was observed in high-risk group patients. The present study has identified a prognostic signature with associated tumor-promoting immune niche with clinical utility in ccRCC. Further exploration of ECM dynamics and validation of this gene signature can assist in design and application of novel therapeutic approaches.

Cancer is one of the leading causes of mortality worldwide among which renal cancer constitutes about 5\% of all cancers in men and $3 \%$ in women with about 140,000 deaths annually worldwide ${ }^{1}$. Renal cancers have very low 5 -year survivability in metastatic renal cell carcinoma $(12 \%)^{2}$. Current prognostic methods are based on clinicopathological features based on TNM (Tumor Node Metastasis staging and grading) which are effective, but the survival of the patients vary even in patients with similar prognosis ${ }^{3}$. The inherent heterogeneity in renal cell carcinoma has been shown to be a primary factor for failure of treatment and development of resistance ${ }^{4}$. Thus, despite several breakthroughs in diagnosis and treatment, high heterogeneity in renal cancer demands further exploration. In this direction, there is still a need to understand the interactions of the molecular players involved in tumorigenesis and its clinical relevance. Extracellular matrix (ECM) is one such entity that forms the biological framework of the tissue and plays a role in its carcinogenesis. ECM is a non-cellular component essential to maintain physical and biochemical stability of the tissue. Its composition varies depending on the tissue and developmental trajectory, but major components are fibrous proteins, proteoglycans, water, and minerals. The bidirectional interaction between cells and ECM influences cell adhesion and migration ${ }^{5}$. ECM is a dynamic entity, and its components are constantly in flux whereby it undergoes deposition and degradation that is significantly altered in diseased conditions. The change in ECM homeostasis has an important role in tumor growth and metastasis. The dynamic nature of ECM influences all the hallmarks of cancer that include uncontrolled growth, angiogenesis, invasion, disruption of metabolomics, and prevention of deadly immune response ${ }^{6}$. 
a.

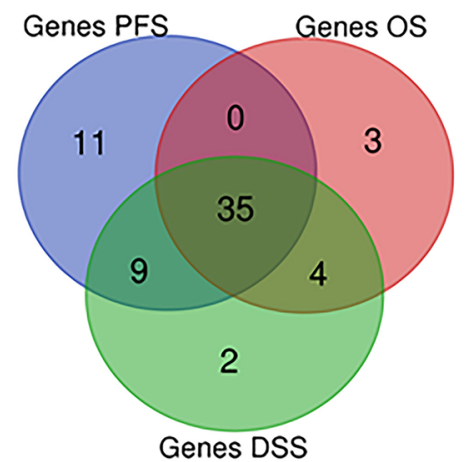

b.

\begin{tabular}{lccc}
\multicolumn{4}{l}{ Prognostically significant genes $(\mathrm{n}=35)$} \\
ITGA3 & MXRA5 & NUDT1 & \\
ANXA2 & WT1 & CCN4 & SERPINE1 \\
GDF10 & LOXL2 & EFNA5 & COL22A1 \\
SBSPON & ADAM12 & GARS & ANXA4 \\
TGM2 & HNRNPA2B1 & ZP3 & SIGLEC1 \\
HSP9OB1 & RARRES2 & SSBP1 & SULF2 \\
P3H2 & LRP1 & SRC & PLOD3 \\
COL3A1 & POSTN & ANXA2P2 & RRAS \\
TNFRSF1A & WNT5B & DNAJB6 & \\
& & &
\end{tabular}

Figure 1. (a) Venn diagram of survival-related ECM genes in renal cancer in TCGA dataset $(\mathrm{z}$ score $>2)$. A total of 35 genes showed significant association with PFS, OS and DSS. (b) List of overlapping 35 genes that showed a significant association with survival. (patients with $\mathrm{z}>2$ score, $p<0.05$ ).

Tumor microenvironment (TME) is a surrounding area of tumor composed of immune cells, blood vessels, cytokines, chemokines, other signalling molecules and $\mathrm{ECM}^{7}$. Along with malignant cells, other cells like endothelial cells, fibroblasts, immune cells interact in ECM in cancer. Several cells like T-regulatory cells, Myeloid-derived suppressor cells (MDSCs), Tumor-associated macrophages (TAMs) create an immunosuppressive environment that is conducive for tumor growth. CD8 T cells, CD4 T-cells, DCs and matrix remodelling enzymes like MMPs and ADAMTs play an important role in maintaining pro-inflammatory immunogenic environment ${ }^{8}$. These variations are important to understand as they are known to play a critical role in tumor growth and invasion ${ }^{9}$. Therefore, the interaction of immune cells and tumor growth and its relevance to ECM is an interesting area of exploration.

The advent of genomics and transcriptomics has led to identification of several gene signatures with prognostic utility in several cancers ${ }^{10-12}$. Here, we explored variation in the expression of extracellular matrix genes in renal clear cell carcinoma. In this study, we developed a 12-gene ECM gene prognostic signature based on TCGA dataset and validated it in 3 independent GEO datasets. These genes had significantly higher expression levels in RCC tumor compared to normal tissue. Further, differential gene analysis identified significant dysregulation of homeostatic functions in high-risk patients. The patients with high ECM signature also showed a higher fraction of tumor-promoting T regulatory and M2 macrophages. These results suggest that the 12-gene ECM signature can be used for prognosis as well as identify patients with suppressive immune phenotypes which can benefit from emerging therapies under research such as T-reg depletion immunotherapies.

\section{Results}

Identification of survival-associated ECM genes. The survival analysis using cBioportal identified 106 ECM genes with higher prevalence in TCGA-KIRC patients ( $\mathrm{z}$-score $>2$, prevalence $>5 \%$ of KIRC dataset $(\mathrm{n}=510)$ (supp. Table 2). Among these 35 genes showed significant association with PFS, OS, and DSS (Fig. 1a,b and supp. Table 3 ). Out of these, 12 genes showed higher expression in ccRCC (Clear cell renal cell carcinoma) tumor compared to normal tissues (Fig. 2a-l). The gene products of these genes are found majorly in extra-cellular compartment and play an oncogenic role in several tumors (Table 1). Univariate and KM analysis identified patients with $\mathrm{z}>2$ score showed a significant association with a higher risk of mortality (Fig. 3a-1). Among these genes, NUDT1 gene showed the highest risk with (H.R 2.56, 95\% CI $1.60-4.10, p<0.01^{\star}$ ).

Development of ECM signature with clinical application and its validation. The TCGA-KIRC cancer cohort $(\mathrm{n}=510)$ was used to assess risk based on Cox proportional hazard model. The survival risk scores were split at median (cut-off $=1.34$ ) to divide patients into low-risk group and high-risk group ( range: 0.18 to 5.90 ). In univariate Cox proportion hazard analysis, the high-risk group was associated with worse survival (H.R 2.45, 95\% CI 1.78-2.45, $p<0.01^{*}$ ). Other significant variables associated with poorer survival were higher age (H.R 1.83, 95\% CI $1.34-2.50, p<0.01^{\star}$ ), advanced stage (H.R 3.2, 95\% CI $2.34-4.37, p<0.01^{\star}$ ), lymph node involvement H.R 3.34, 95\% CI $1.77-6.34, p<0.01^{*}$ ), and metastasis (H.R 4.36, 95\% CI 3.18 $5.98, p<0.01^{\star}$ ) (Fig. 4a). In multivariate Cox proportion hazard only four variables showed association with poorer survival:-Higher risk (H.R 2.05, 95\% CI 1.27-3.28, $p<0.01^{\star}$ ), higher age (H.R 1.77, 95\% CI $1.15-2.72$, $p<0.01^{*}$ ), advanced stage (H.R 2, 95\% CI $1.26-3.19, p<0.01^{*}$ ), and metastasis (H.R 2.83, 95\% CI $1.76-4.55$, $p<0.01^{*}$ ) (Fig. 4b). Further, KM analysis significantly differentiated patients based on overall survival (Fig. 5a).

For external validation, this 12 gene signature was validated in 3 independent GEO datasets. GSE3538 (177 patients) $\mathrm{HR}=2.2 ; 95 \%$ CI [1.42-3.42; $p<0.01^{*}$, GSE33371 (23 patients) $\mathrm{HR}=17.44 ; 95 \%$ CI [3.61-84.32; $p<0.01^{\star}$, GSE22541 (24 patients) HR $=4.54 ; 95 \%$ CI [1.53-13.46]; $p<0.05^{*}$ (Fig. 5b-d). $\chi 2$ test between high and low risk categorical variables found significant association with ethnicity, stage, metastasis, aneuploidy score, fraction genome altered, OS status, PFS status and DSS status (Table 2). 

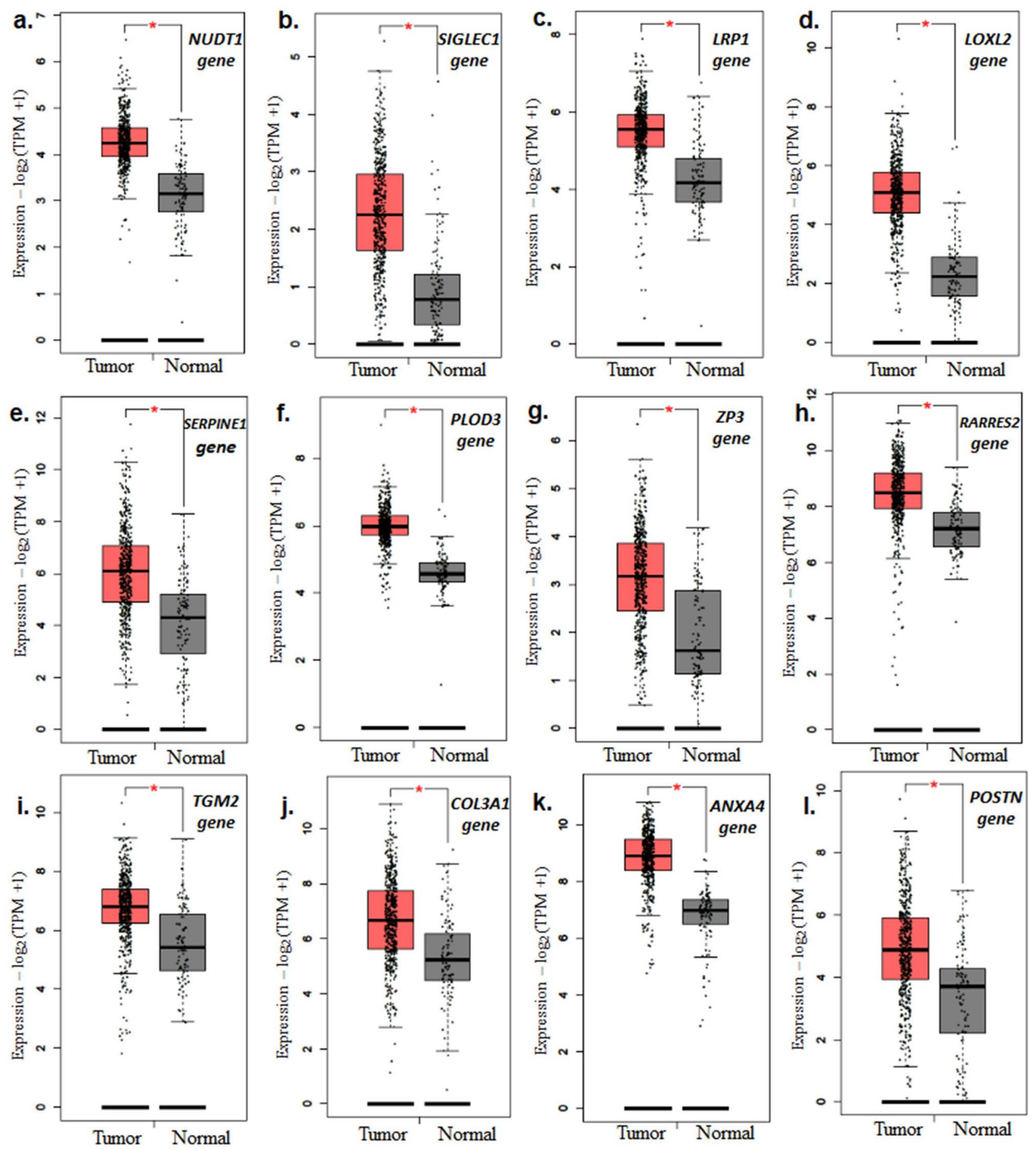

Figure 2. 12 genes with higher expression in tumor (523 patients) compared to normal tissue (100 tissue). (a) NUDT1, (b) SIGLEC1, (c) LRP1, (d) LOXL2, (e) SERPINE1, (f) PLOD3, (g) ZP3, (h) RARRES2, (i) TGM2, (j) COL3A1, (k) ANXA4, and (1) POSTN.

Differentially expressed genes (DEGs) in high and low-risk patients. DEseq2 analysis was performed to screen differentially expressed genes between high and low-risk patients' group (supp Table 4). Among 20,501 protein-coding genes, 1818 genes were upregulated at $>$ twofold in high-risk patients compared to lower risk group. In the low-risk group, a total of 159 genes were upregulated at > twofold compared to the high-risk group (supp Table 4). Principal component analysis (PCA) showed a clear separation between the high-risk group and low-risk group based on ECM signature (Fig. 6a). Volcano plot of differentially expressed genes between high-risk and low-risk patients is depicted in Fig. 6b. The enriched gene ontology terms in high ECM group were extracellular matrix structural constituent and acute phase response pathways (Fig. 6c). The enriched gene ontology terms in low ECM group were transmembrane transporter activity and transport (Fig. 6d). Fur- 


\begin{tabular}{|c|c|c|c|}
\hline Gene symbol & ECM conf. value & Role in tumorigenesis & References \\
\hline $\begin{array}{l}\text { NUDT1 } \\
\text { (Nudix Hydrolase 1) }\end{array}$ & 3 & $\begin{array}{l}\text { NUDT1 enzyme removes oxidized nucleotide pools and prevents its subsequent misincor- } \\
\text { poration into DNA. Its higher expression has been associated with several cancers includ- } \\
\text { ing renal cancer, brain, lung, and liver cancer }\end{array}$ & 13,14 \\
\hline $\begin{array}{l}\text { SIGLEC1 } \\
\text { (Sialic Acid Binding Ig Like Lectin 1) }\end{array}$ & 5 & $\begin{array}{l}\text { SIGLEC1 expressing cells, like macrophages provide anchorage for metastatic cells. } \\
\text { SIGLEC1 overexpression has been associated with poorer survival in several cancers }\end{array}$ & 15,16 \\
\hline $\begin{array}{l}\text { LRP1 } \\
\text { (LDL Receptor Related Protein) }\end{array}$ & 3 & $\begin{array}{l}\text { LRP1 plays a significant role in the endocytosis of lipoproteins. LRP1 overexpression has } \\
\text { been associated with invasion and migration of tumor cells }\end{array}$ & 17,18 \\
\hline $\begin{array}{l}\text { LOXL2 } \\
\text { (Lysyl Oxidase Like 2) }\end{array}$ & 5 & $\begin{array}{l}\text { LOXL2 is part of extracellular enzyme family which cross-links elastin and fibrillar col- } \\
\text { lagen. LOXL2 overexpression has been associated with several cancers including breast, } \\
\text { gastric, colon, esophageal and pancreatic cancer }\end{array}$ & 19 \\
\hline $\begin{array}{l}\text { SERPINE1 } \\
\text { (Serpin Family E Member 1) }\end{array}$ & 5 & $\begin{array}{l}\text { SERPINE1 is an inhibitor of urokinase and tissue plasminogen activator. It is associated } \\
\text { with poorer prognosis in several cancer including stomach, head and neck, sarcoma, } \\
\text { urothelial and testicular cancer }\end{array}$ & 20 \\
\hline $\begin{array}{l}\text { PLOD3 } \\
\text { (Procollagen-Lysine,2-Oxoglutarate 5-Dioxygenase 3) }\end{array}$ & 5 & $\begin{array}{l}\text { PLOD enzymes perform Lysyl hydroxylation of collagen. PLOD3 also performs glycosyla- } \\
\text { tion activity of collagen hydroxylysines. Higher expression of PLOD3 has been associated } \\
\text { with several cancers }\end{array}$ & 21,22 \\
\hline $\begin{array}{l}\text { ZP3 } \\
\text { (Zona Pellucida Glycoprotein 3) }\end{array}$ & 5 & ZP3 was found to be part of core secretome signature across multiple cancer types & 23 \\
\hline $\begin{array}{l}\text { RARRES2 } \\
\text { (Retinoic Acid Receptor Responder 2) }\end{array}$ & 5 & $\begin{array}{l}\text { RARRES2 is secreted ligand for CMKLR1 expressing immune cells. Its expression is associ- } \\
\text { ated with the chemoattraction of immune cells }\end{array}$ & 24 \\
\hline TGM2 (Transglutaminase 2) & 5 & $\begin{array}{l}\text { TGM2 assists in posttranslational modification of proteins. Its overexpression is involved in } \\
\text { several cancers including pancreatic, prostate, ovarian and breast cancer }\end{array}$ & 25 \\
\hline $\begin{array}{l}\text { COL3A1 } \\
\text { (Collagen Type III Alpha } 1 \text { Chain) }\end{array}$ & 5 & $\begin{array}{l}\text { COL3A1 expresses components of fibrillar collagen which forms the skin and other tissues. } \\
\text { Its over-expression in involved in multiple cancers }\end{array}$ & 26 \\
\hline $\begin{array}{l}\text { ANXA4 } \\
\text { (Annexin A4) }\end{array}$ & 4 & $\begin{array}{l}\text { Annexin A4 is calcium-regulated phospholipid-binding protein. ANXA4 upregulation is } \\
\text { involved in multiple cancers and is a biomarker for drug resistance }\end{array}$ & 27,28 \\
\hline $\begin{array}{l}\text { POSTN } \\
\text { (Periostin) }\end{array}$ & 5 & $\begin{array}{l}\text { POSTN is a multimodular protein which interacts with ECM proteins such as tenascin C, } \\
\text { collagen, and fibronectin. Its expression is deregulated in multiple pathologies including } \\
\text { inflammation and malignant transformation }\end{array}$ & 29 \\
\hline
\end{tabular}

Table 1. Biological role of 12 genes. The ECM confidence value ranged from 0 (absence of any extracellular evidence), 1 (low confidence of extracellular evidence) to 5 (highest confidence of extracellular evidence). Cellular localization of these genes was incorporated from Genecards (https://www.genecards.org/).

ther network analysis using GENEmania portal revealed co-expression based on protein-protein interaction to be high (66.37\%) \& the physical interaction was at $23.81 \%$ (Fig. 6e).

Key pathways associated with high and low-risk patients using Gene Set Enrichment Analysis. GSEA analysis was performed to study two risk clusters in greater resolution. The analysis indicated significant perturbations of molecular pathways in the high-risk group. The pathways with highest normalized enrichment scores (NES) in the low risk ccRCC patients were GO active ion transmembrane transporter $(\mathrm{NES}=-2.4$, $\mathrm{FDR}=<0.001)$, Hallmark fatty acid metabolism gene signature (NES $=-2.1, \mathrm{FDR}=<0.001)$, (Fig. 7a,b). Among other pathways were GO active transmembrane transporter activity (NES $=-2.38$ ), GO secondary active transmembrane transporter activity $(\mathrm{NES}=-2.27)$ and Hallmark oxidative phosphorylation $(\mathrm{NES}=-2.07)$ (suppl tables 5,6). Interestingly, patients in low risk were enriched in transportation-related kidney pathways, whereas these homeostatic pathways were absent in high-risk patients which included diverse pathways with low NES such as Extracellular matrix $(\mathrm{NES}=0.64)$, Hallmark Epithelial-mesenchymal transition $(\mathrm{NES}=0.69)$ and Hallmark allograft rejection $(\mathrm{NES}=0.63)$.

Correlation analysis of ECM gene signature and infiltration of immune cells. To analyze the distribution of immune cells in these two risk groups, CIBERSORT-ABS algorithm was utilized (suppl Fig. 1). It identified patients in high risk with higher abundance of T regulatory cells, M0 and M2 macrophages (Fig. 7c). Although tumor-promoting immune features were found to be correlated with the high-risk group there was no association of inflammatory immune cells with any group (suppl Fig. 2). The constellation plot showed 2 distinct clusters depicting high-risk ECM rich patients and low-risk patients (Fig. 7d).

\section{Discussion}

Extracellular matrix (ECM) has been commonly viewed as inert scaffolding for growth and development of tissues $^{30}$. This view is rapidly being revised due to its emerging active roles in health and disease ${ }^{31}$. ECM plays an important role in maintaining structural integrity and amplification of growth signals by providing binding sites for various growth factors. For instance, FGF (fibroblast growth factors) and VEGF (vascular endothelial growth factors) have dual binding sites for cell adhesion and growth factors ${ }^{32}$. This localization of growth factors plays a critical role in the maintenance of gradients, which is essential in cell signalling. Further, the extracellular matrix and its perturbation plays a significant role in tumor invasion and metastasis especially in solid cancer $^{31}$. In cancer, epithelial cells can remodel ECM to pro-tumorigenic tumor microenvironment through activation of stromal cells ${ }^{33}$. This transition of normal ECM due to altered expression of ECM genes can assist 
a.

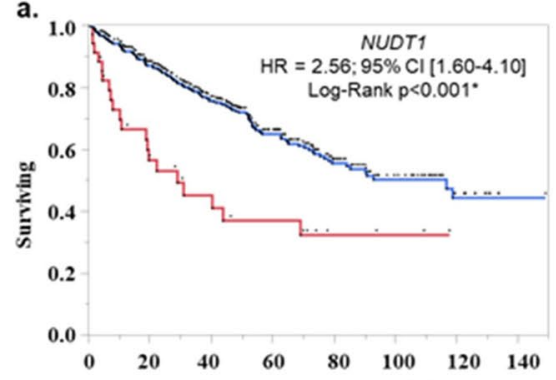

d.

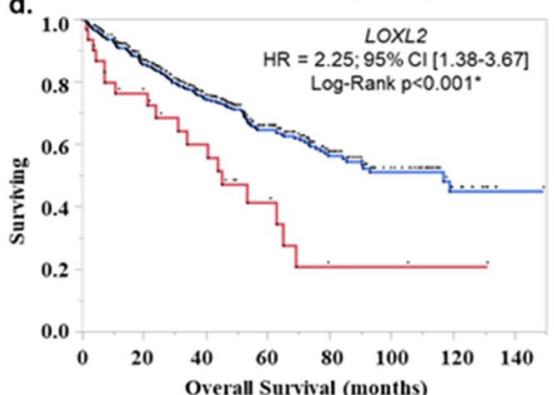

g.

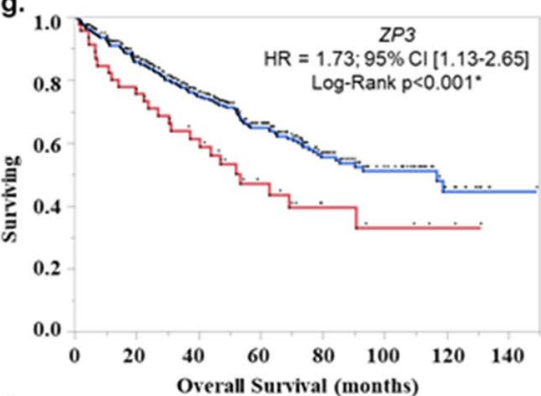

j.

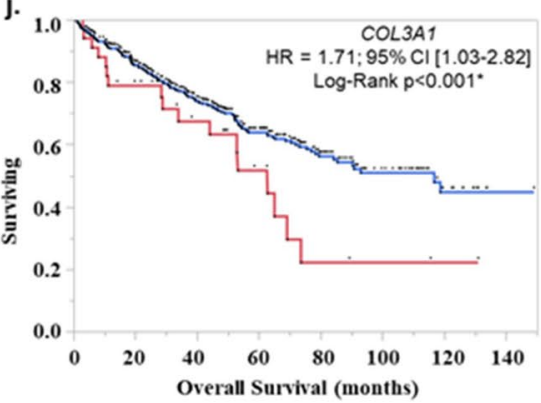

b.

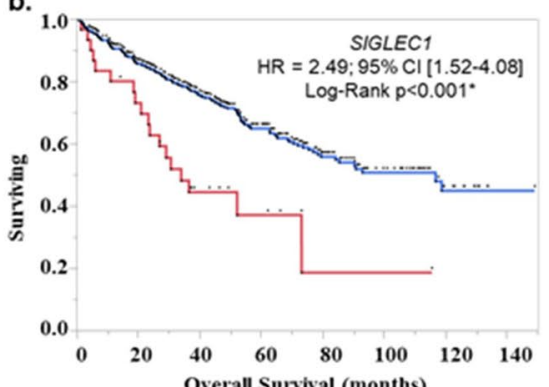

e.

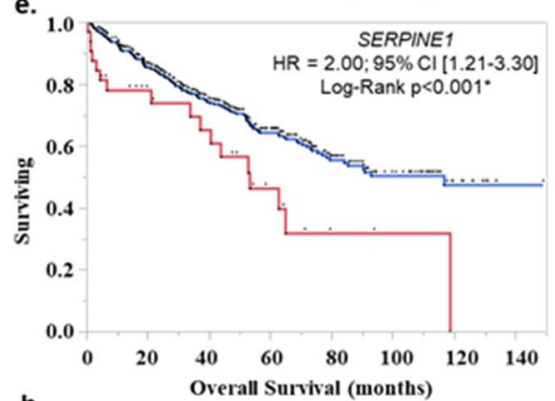

h.

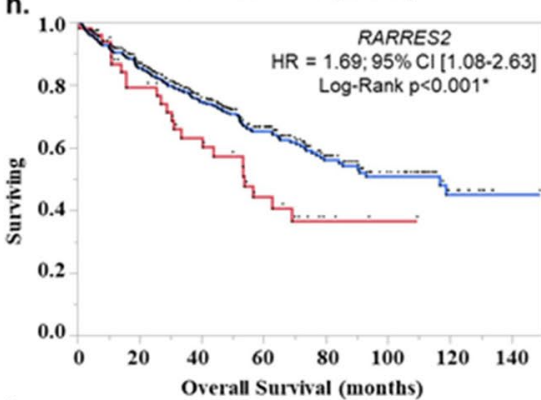

k.

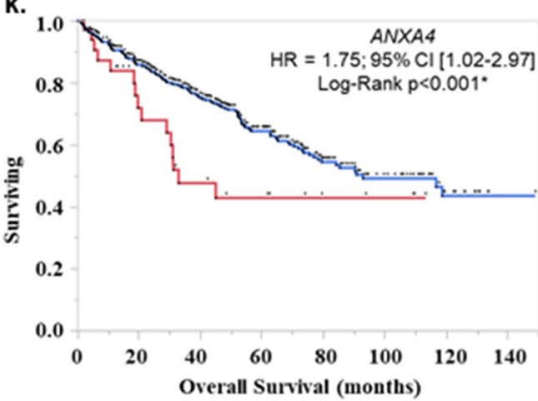

c.

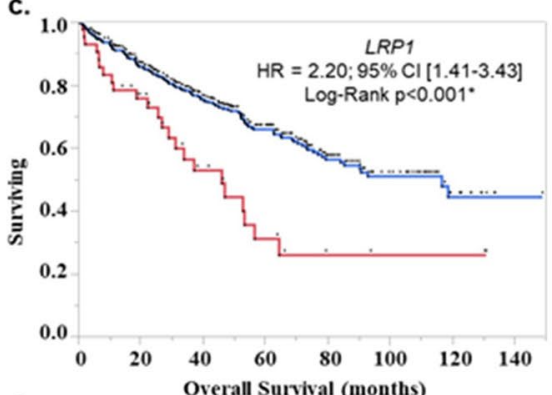

f.

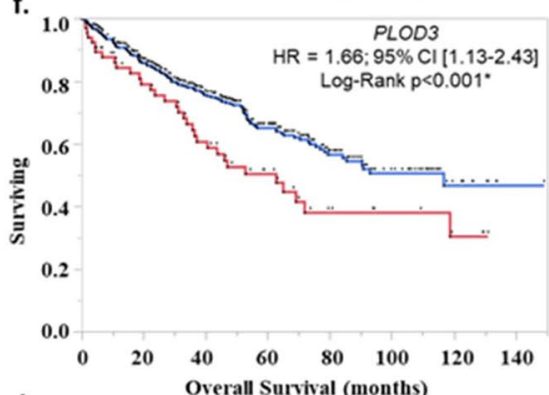

i.

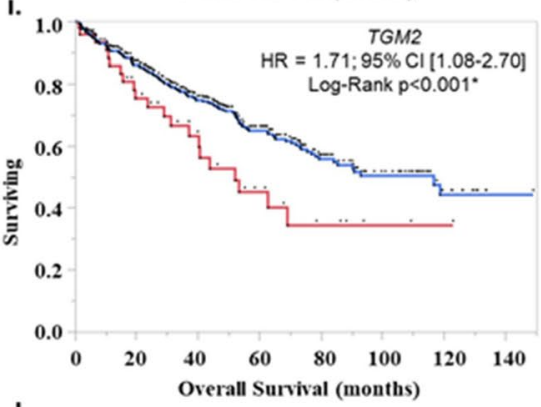

I.

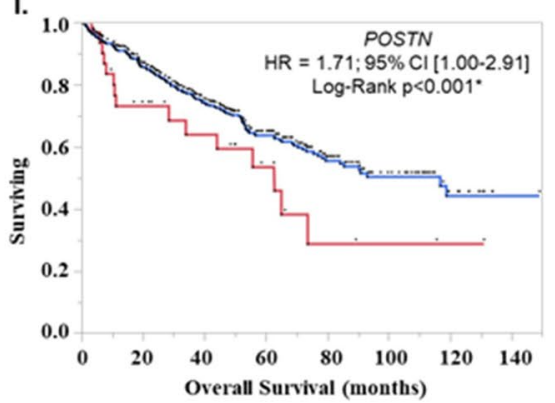

High Risk Low Risk

Figure 3. KM analysis of ECM genes with higher expression ( $\mathrm{z}>2$ score) in RCC patients. (a) NUDT1, (b) SIGLEC1, (c) LRP1, (d) LOXL2, (e) SERPINE1, (f) PLOD3, (g) ZP3, (h) RARRES2, (i) TGM2, (j) COL3A1, (k) ANXA4, and (l) POSTN. The patients with higher expression (cutoff: $\mathrm{z}$-score $>2$ ) were grouped in high risk and other patients were in the low-risk group.

in prognostication of renal cancer. In the present study, the expression of ECM-related genes was explored to develop a prognostic signature using TCGA and other independent gene expression datasets.

Apart from the critical role of ECM plasticity in the spread of cells and its malignant transformation, it has important immunological implications as well ${ }^{34,35}$. It has been shown that ECM can negatively affect the infiltration and distribution of immune cells such as CD8 + T cells and NK cells in solid tumors ${ }^{36,37}$. Abnormal ECM components can alter mechanical and biochemical properties of TME which can affect immune cells function ${ }^{38}$. Additionally, immunosuppressive molecules such as IL-10 and TGF- $\beta$ accumulate in ECM rich tumor partly due to low diffusion and buildup of hypoxic and metabolic stress ${ }^{37,39}$. The deposition of ECM components can lead to accumulation of immunosuppressive T-regulatory cells and M2 polarized macrophages ${ }^{8}$. Thus, variations in ECM components can affect distribution, activation, and polarization of immune cells.

In the current study, we explored the prognostic potential of genes related to ECM remodelling and its correlation to immune cell infiltration in ccRCC. We have identified $12 \mathrm{ECM}$ gene signatures in ccRCC with 
a.

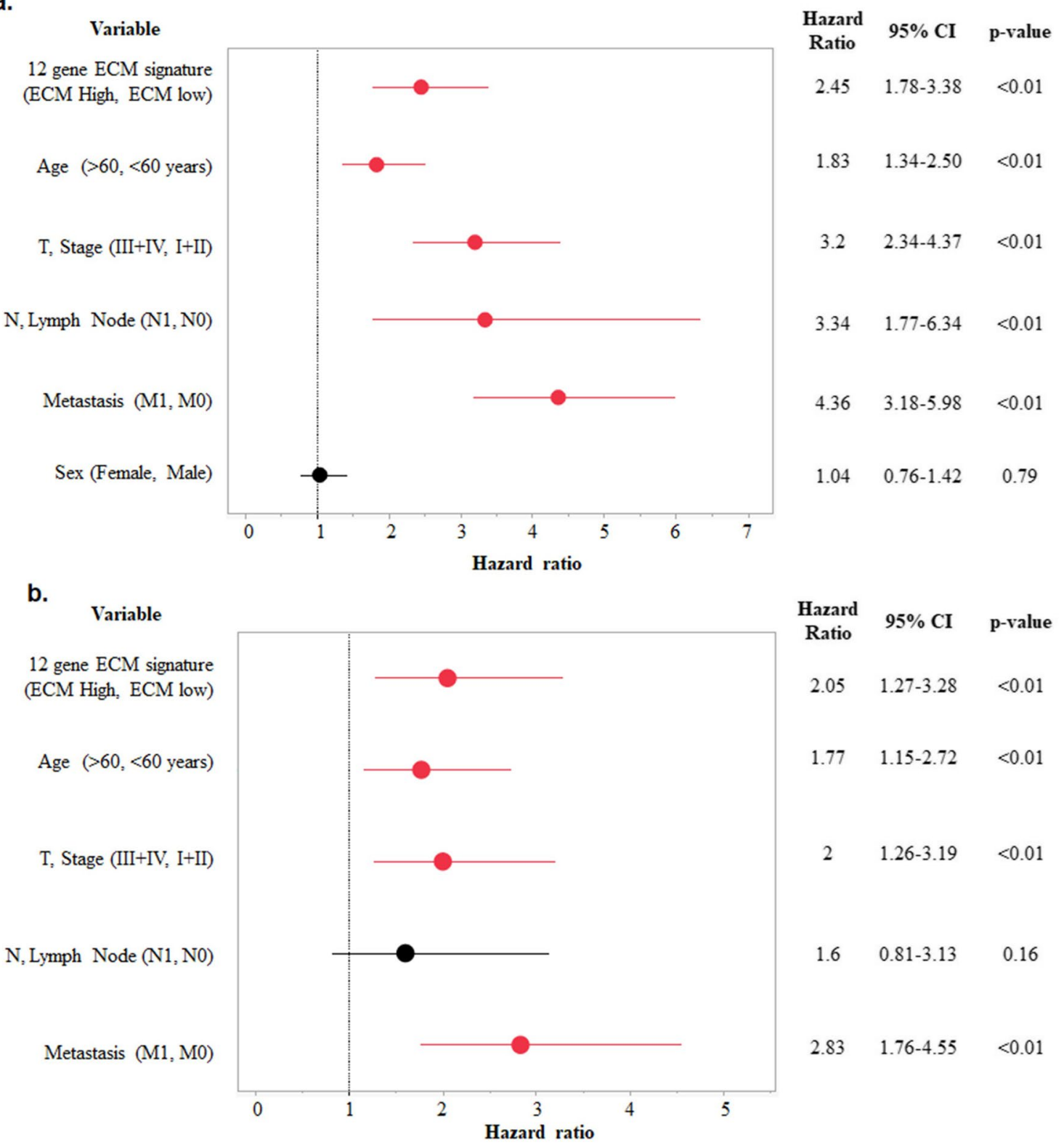

Figure 4. (a) Univariate and (b) Multivariate cox regression analysis of 12 gene ECM signature with age, Stage, $\mathrm{N}$, Metastasis, and sex.

prognostic significance in multiple datasets. Before this study, a comprehensive analysis of ECM genes to identify prognostic gene signature and its immune characterization was lacking. In this study, we have addressed the issue by exploring $964 \mathrm{ECM}$ related genes and developed a 12 gene prognostic signature which was derived from TCGA and validated using independent GEO datasets. Further, these 12 genes were found to be tumorigenic as their expression levels were found to be significantly higher levels in renal cancer compared to normal tissue. Secretion of ECM modifying enzyme families such as LOX, PLOD, and collagen among others can lead to higher invasiveness, migration, proliferation, and survival of cancer cells ${ }^{35}$. The protein products of this gene signature are pre-dominantly secreted in extra-cellular spaces and play tumorigenic role. Further, network analysis of this prognostic disease module identified higher co-expression of these genes. The protein co-expression networks are generally used to identify genes which are functionally relevant and are under similar transcriptional program ${ }^{40}$. The expression level of these genes has been found to be similar across several conditions which is indicated by its higher co-expression (>66.37\%). In a separate study, high ECM gene expression was found to be prognostic for breast cancer ${ }^{41}$ and gastric cancer ${ }^{42}$. Interestingly one of the studies has identified correlation of aggressive ECM characteristics with immunosuppressive features in glioblastoma ${ }^{43}$. In renal cancer, gene like collagen 1 has been explored in enhanced metastasis and invasion in $\mathrm{RCC}^{44}$. Another study has identified a higher expression of 10 genes associated with cell adhesion and ECM regulation in renal cancer ${ }^{45}$.

In GSEA analysis, the patients in the high-risk group showed significant perturbation in normal homeostasis kidney function such as active ion transportation and fatty acid metabolism. These functions are known to be 
a.

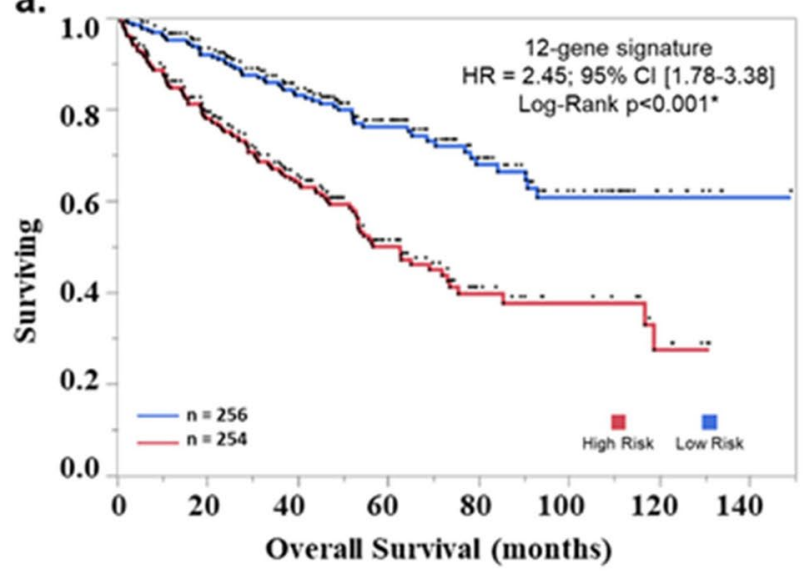

b. GSE3538

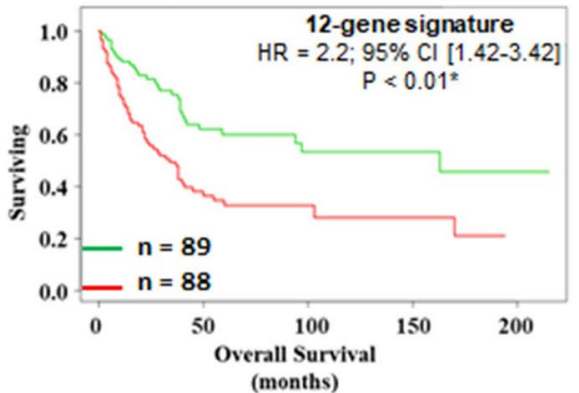

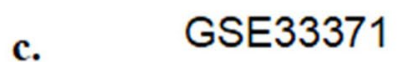

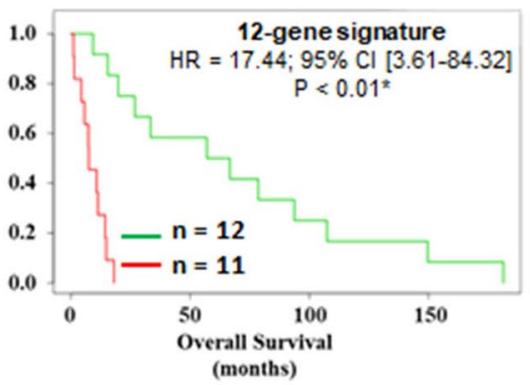

d.

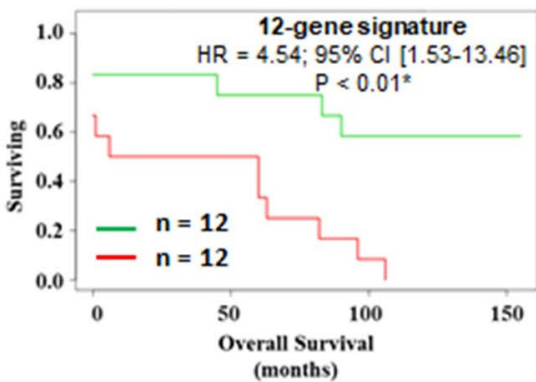

High Risk Low Risk

Figure 5. Survival analysis of 12 gene signature with kidney renal cell cancer patients in (a) TCGA dataset, (b) GSE3538, (c) GSE33371, and (d) GSE22541. Patients were divided using median risk-score value as cut-off dividing in high-risk and low-risk subgroups.

\begin{tabular}{|c|c|c|c|c|c|c|c|}
\hline Clinical parameter & High ECM $(n=255)$ & Low ECM $(n=255)$ & Pearson $\chi^{2} \mathbf{p}$-value & Clinical parameter & High ECM $(n=255)$ & Low ECM $(\mathbf{n}=255)$ & Pearson $\chi^{2} \mathbf{p}$-value \\
\hline Age & & & 1 & $\begin{array}{l}\text { Fraction genome } \\
\text { altered }\end{array}$ & & & $<0.01^{*}$ \\
\hline$<66$ years & 128 & 128 & & $<0.120$ & 108 & 150 & \\
\hline$>66$ years & 127 & 127 & & $>0.120$ & 147 & 105 & \\
\hline Ethnicity & & & $<0.01^{*}$ & OS & & & 0.05 \\
\hline African American & 36 & 19 & & $<5$ years & 191 & 171 & \\
\hline Caucasian & 213 & 227 & & $>5$ years & 64 & 84 & \\
\hline Sex & & & 0.51 & OS status & & & $<0.01^{*}$ \\
\hline Female & 89 & 96 & & Living & 150 & 192 & \\
\hline Male & 166 & 159 & & Deceased & 105 & 63 & \\
\hline Stage & & & $<0.01^{*}$ & PFS & & & $<0.02$ \\
\hline $\mathrm{I}+\mathrm{II}$ & 132 & 171 & & $<5$ years & 206 & 185 & \\
\hline $\mathrm{III}+\mathrm{IV}$ & 123 & 84 & & $>5$ years & 49 & 70 & \\
\hline Lymph Node & & & 0.059 & PFS Status & & & $<0.01^{*}$ \\
\hline No & 117 & 111 & & No progression & 153 & 199 & \\
\hline N1 & 12 & 4 & & Progression & 102 & 56 & \\
\hline Metastasis & & & $<0.01^{*}$ & DSS & & & 0.05 \\
\hline No Metastasis M0 & 188 & 213 & & $<5$ years & 191 & 171 & \\
\hline Metastasis M1 & 51 & 27 & & $>5$ years & 64 & 84 & \\
\hline Aneuploidy score & & & $<0.01^{*}$ & DSS status & & & $<0.01^{*}$ \\
\hline$>4$ & 143 & 81 & & Tumor free & 172 & 221 & \\
\hline$<4$ & 112 & 174 & & Dead with tumor & 76 & 31 & \\
\hline
\end{tabular}

Table 2. Comparison of clinicopathological features of patients with enriched ECM and low ECM gene signature using Pearson's chi-square analysis (TCGA-KIRC dataset). 
a.

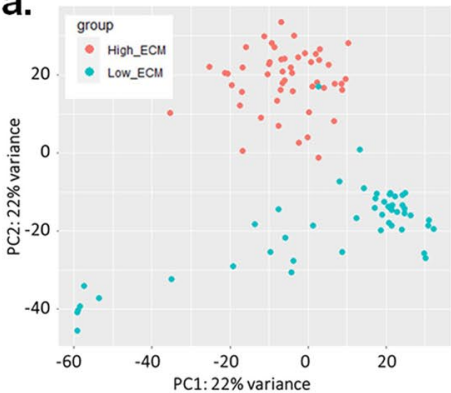

b.

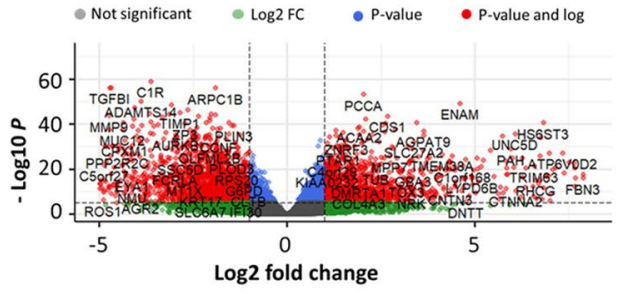

c.

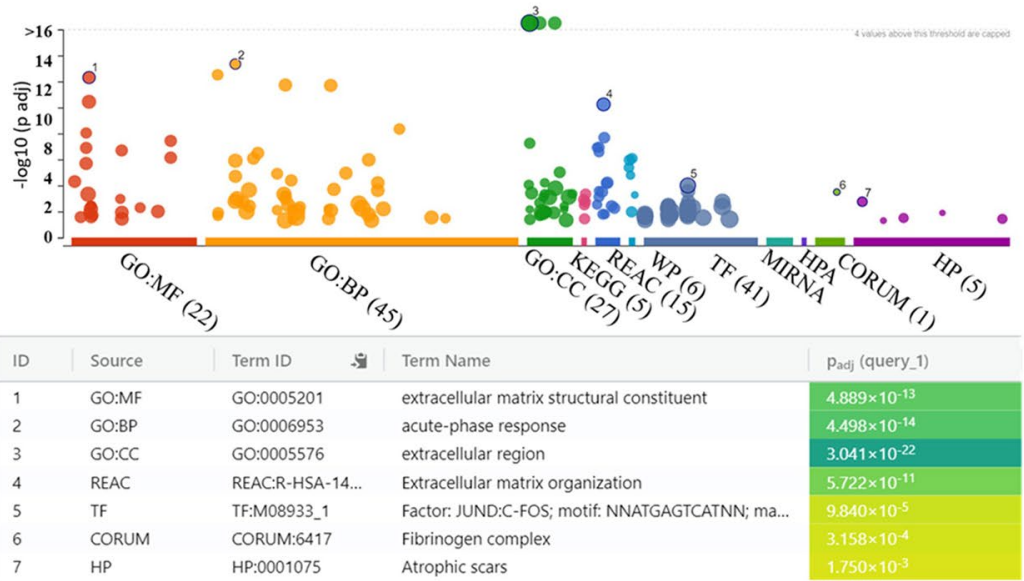

d.

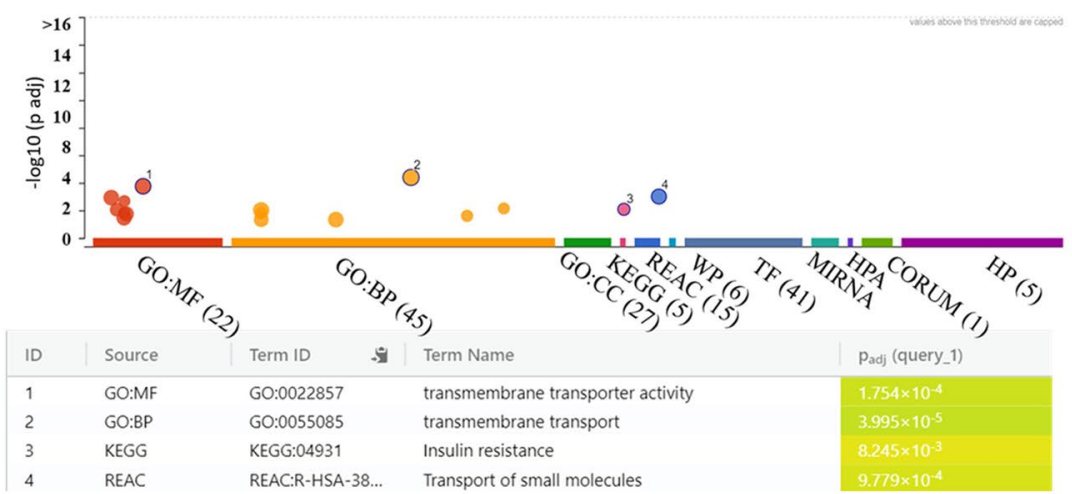

e.

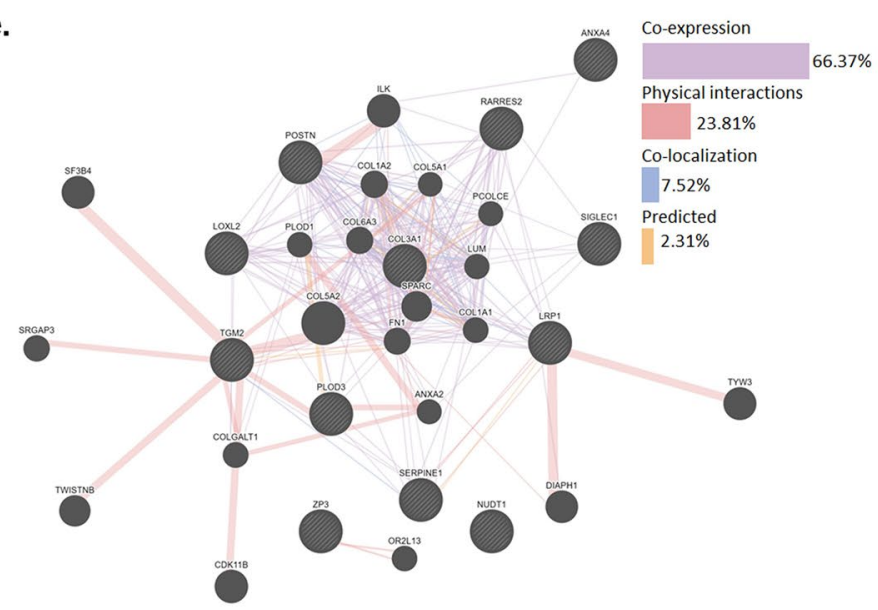

Figure 6. (a) Principal component analysis (PCA) showing distinct clustering between RNA-seq expression of high CDI patients (topmost 50 patients) compared to low CDI patients (bottom 50 patients). (b) Volcano plot showing differential expression of genes between High CDI and low CDI $(p<0.01, \log 2$ fold-change $>2)$. (c) Functional enrichment analysis of highly expressed genes ( $\log 2$ fold-change $>2)$ in $(\mathbf{c})$. High ECM group with most hits for extracellular matrix and (d) Low ECM group with maximum hits for transmembrane transport activity. (e) GeneMANIA gene-gene interaction network showed multiple connections, pre-dominantly co-expression and physical interaction among genes in prognostic signature. 
a. GO_ACTIVE_ION_TRANSMEMBRANE_TRANSPORTER_A CTIVITY

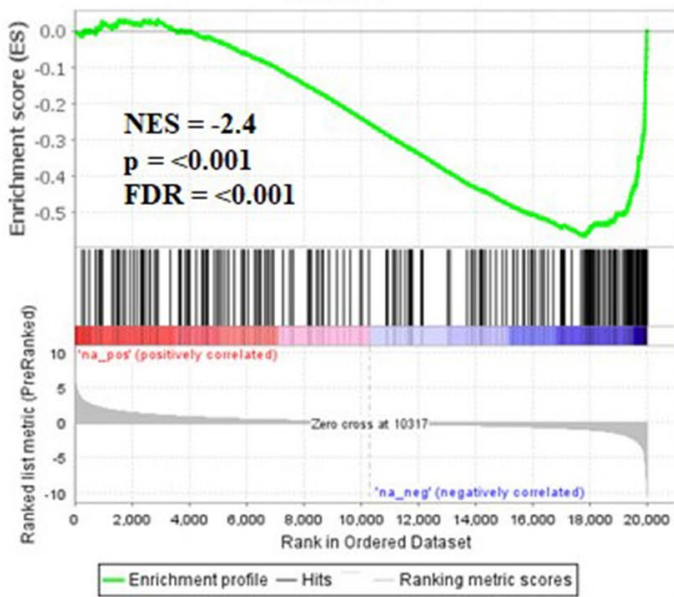

C.

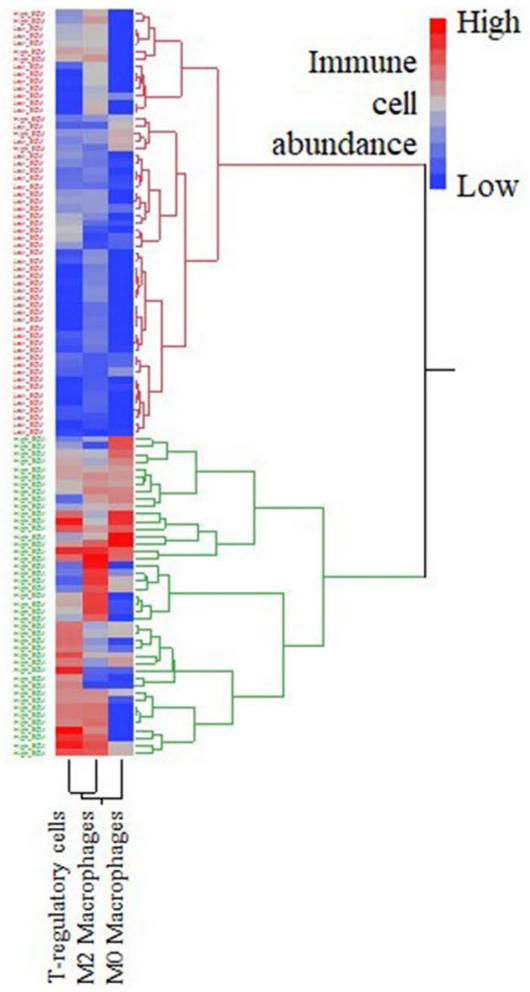

b. Enrichment plot: HALLMARK_FATTY_ACID_METABOLISM

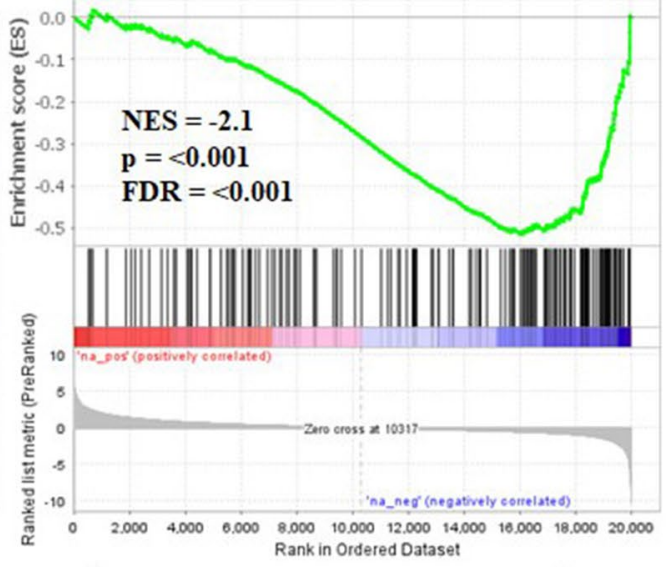

d. -Enrichment profile — Hits - Ranking metric scores

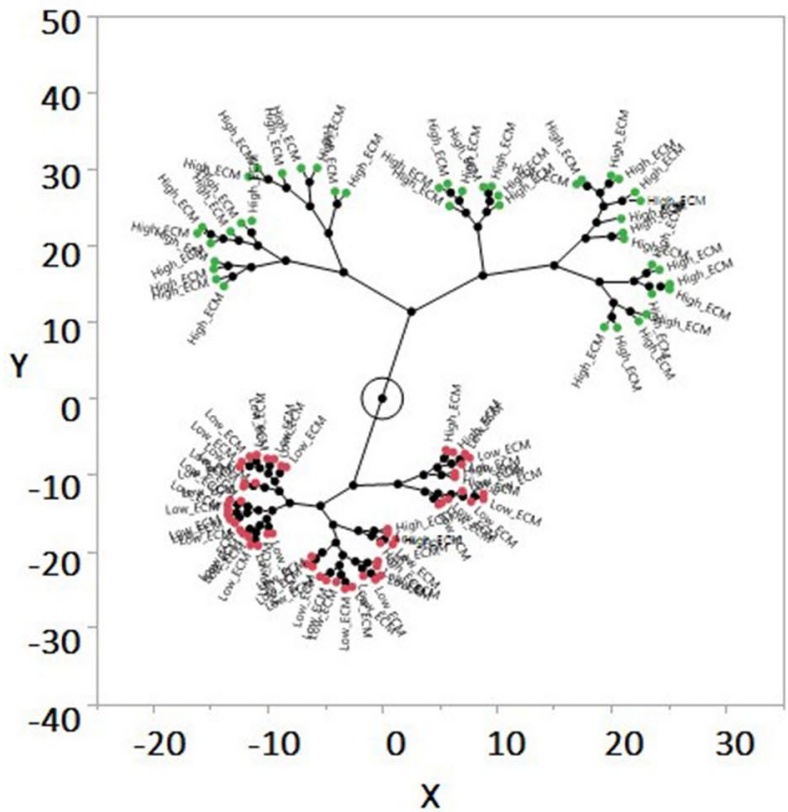

Figure 7. GSEA analysis of the differentially expressed genes between the two risk groups. (a) Pathways enriched in the low-risk group were (b). Active ion transmembrane transporter and (b). Hallmark of fatty acid metabolism. (c) Hierarchical tree structure and (d) constellation plot classifying the high-risk patients according to correlation with tumor-promoting immune cell infiltration.

associated with kidney functions and renal epithelial cells utilize fatty acids are the source of energ ${ }^{46}$. One of the striking features in low-risk group was the preservation of kidney function genes. Chronic kidney disease (CKD) and lower estimated glomerular filtration rate (eGFR) is associated with increased risk of renal cancer ${ }^{47}$. Further, the clinical management of ccRCC patients includes maximization of kidney function preservation and management of long-term $\mathrm{CKD}^{48}$. This gene signature identified patients with high ECM at a higher risk of renal complications compared to the low-risk patient group.

Additional analysis of immune abundance using CIBERSORT-ABS revealed that T-regulatory, M2 macrophages and M0 macrophages were found to be higher in high-risk patients. In tumor microenvironment, T-reg and suppression of the immune system plays a significant role in progression of cancer ${ }^{49}$. Recently, high infiltration of M2 macrophages and FOXP3 + T-regulatory cells was associated with adverse clinical outcome 
in renal carcinoma ${ }^{50}$. The combination of anti-CTLA- 4 and anti-PD- 1 in a CheckMate 214 trial, was found to achieve objective response rate in only $40 \%$ of patients ${ }^{51}$. In our study, the association of M2 macrophages and $\mathrm{T}$ regulatory cells in ECM high-risk patients represent a risk population which might not benefit from these immunotherapies. These patients can be targeted for emerging novel therapeutic approaches currently under investigation such as T-reg depletion strategies or anti-FoxP3 Treg vaccines ${ }^{52}$.

Further, expanded knowledge of variables in ECM distribution can illuminate 2 critical aspects of novel immunotherapeutic approaches: its design and application. First, a recent study has identified that targeted blocking of ECM molecules can lead to a response in immune-refractive patients. In this study, integrin $\alpha v \beta 6 / 8$ specific monoclonal antibody $(\mathrm{mAb})$ led to higher survival in TNBC metastatic mouse models which responded poorly to PD-1 blockade ${ }^{53}$. So, direct inhibition of specific ECM components can lead to higher survival in cancer models. Secondly, understanding of physical and biochemical constraints posed by ECM can assist in generating better ways to deliver novel drugs to the tumors. Abnormal ECM matrix leads to poor diffusion of drugs, nutritional supply, and immune mediators ${ }^{37}$. Active ECM modulation has shown promising response to the efficacy of chemotherapeutic regimes in various models. Inhibition of LOX gene led to an improved response to chemotherapy in tumor models ${ }^{54}$. Comparatively, for an effective response to an immunotherapy regime, there is an addition of another variable i.e., immune cells. Immune modulators (mAbs) and immune cells are required to be in proximity with tumor for an effective response, but the ECM is known to interfere with both. Physically, ECM prevents interaction between immune cells such as T cells and tumor cells ${ }^{55}$. Further, ECM also negatively affects the diffusion of immunotherapy molecules in tissues. Dense, highly cross-linked, and stiff ECM matrix interferes with the diffusion of immunomodulatory drugs like ipilimumab (anti-CTLA-4) due to their larger hydrodynamic diameter ${ }^{37}$. Further, novel ECM-targeting drugs such as collagenase-infused nanogels can penetrate ECM-rich tumors and could be explored for such therapies ${ }^{56}$. Thus, further understanding of survival-related ECM genes in ccRCC and its effect on immune cells can assist in the design and application of novel therapeutics.

\section{Conclusion}

In the current study, we developed a 12-ECM gene prognostic signature which was able to discriminate high-risk renal cell carcinoma patients from low-risk ones. Besides, this signature was validated in 3 independent datasets. Moreover, the tumorigenic role of the genes included in this signature showed elevated expression in tumor tissues compared to normal. PCA and differential gene expression analysis identified significant perturbation of normal homeostatic functions of high-risk patients. Further, immune-suppressive cells such as T-reg, M2 and M0 macrophages showed higher infiltration in high-risk patients. The prognostic signature and immune features can identify important sub-section of ccRCC patients which can benefit from emerging immunotherapies.

\section{Methods}

Data acquisition and pre-processing. A total of 964 extracellular matrix and remodeling genes were downloaded from Gene Ontology (http://geneontology.org) ${ }^{57}$ (suppl. Table 1). The gene list was curated based on range of evidence for their involvement in the ECM pathway. The evidence annotation of these genes included were: 'Inferred from Experiment (EXP)', 'Inferred from Direct Assay (IDA)', 'Inferred from Physical Interaction (IPI)', 'Inferred from Mutant Phenotype (IMP)', 'Inferred from Genetic Interaction (IGI)' and 'Inferred from Expression Pattern (IEP)' (http://geneontology.org/docs/guide-go-evidence-codes/). This approach led to inclusion of only specific genes with evidence of their involvement in ECM interaction pathways. Transcriptome RNA-sequencing and clinical data of kidney renal cell carcinoma cancer (KIRC) patients $(n=510)$ was extracted from the TCGA data portal (https://portal.gdc.cancer.gov/). This dataset was used for GSEA analysis, immune cell infiltration analysis and clustering analysis. The clinical information of patients and $\mathrm{z}$-scores of individual genes was downloaded from cBioportal (https://www.cbioportal.org $/)^{58}$. The z-scores were divided into categorical variables ( $\mathrm{z}$-score $>2$ and $\mathrm{z}$-score $<2$ ) for survival analysis. A total of $523 \mathrm{ccRCC}$ cancer patients and 100 normal kidney tissues from GTEx portal was compared using GEPIA portal (http://gepia2.cancer-pku.cn/) ${ }^{59}$. GEO (Gene expression Omnibus) datasets (GSE3538, n=177, GSE33371, $\mathrm{n}=23$ and GSE22541, n=24) were explored for validation of prognostic signature ${ }^{60}$.

Identification of survival-associated ECM genes. 964 extracellular matrix and remodeling genes were analyzed in cBioportal to quantify perturbations in TCGA-renal cell carcinoma (TCGA-KIRC RNA Seq V2, $\mathrm{n}=510$ patients) based on $\mathrm{z}$ score more than 2 as has been used previously to screen prognostic genes ${ }^{61}$.

Comparison of prognostic-related genes between tumor and normal tissue. The expression of the genes associated with overall survival (OS) of ECM genes in ccRCC were compared to normal tissue using Gene Expression Profiling Interactive Analysis. For this study, the Kidney tumor $(n=523)$ dataset was compared against combined gene expression data of normal tissues from TCGA and Genotype-Tissue Expression (GTEx) data $(n=100)$, respectively. In GEPIA, parameters with Log2FC (Fold change) cutoff were set at 1 with a $p$-value cut-off at 0.01 .

Development of 12 gene ECM signature. Independent prognostic potential of 12 genes was analyzed in renal cancer (TCGA-KIRC-cBioportal) based on log-rank p-values. A total of 510 patients with RNA-Seq data were included in this analysis. KM curves with overall survival (OS) and Progression-free survival (DFS) were generated. OS shows total duration for which patients were alive from date of diagnosis. To develop a 12 gene signature, Cox regression model was developed that divided the cohort into low- and high-risk groups ECM risk score based on median as previously published ${ }^{62-64}$. Briefly, the prognostic score was calculated by 
multiplying the expression value of a gene with its corresponding Cox proportion regression coefficient (Prognostic score $=\Sigma$ Cox regression coefficient of $\mathrm{Gene}_{\mathrm{i}}{ }^{*}$ expression value of gene $\mathrm{Gene}_{\mathrm{i}}$ ). For validation of gene signature, GEO (Gene expression Omnibus) datasets were explored GSE3538 $(n=177)$, GSE33371 $(n=23)$ and GSE22541 ( $\mathrm{n}=24)$ using SurvExpress tool (http://bioinformatica.mty.itesm.mx:8080/BiomatecSurvivaX.jsp). The microarray expression data used in these studies were acquired from Affymetrix Human Genome array [HG-U133_Plus_2, GPL platform].

Biological role of $\mathbf{1 2}$ genes. To further evaluate the biological roles of 12 genes and analyze predominant cellular compartment of their expressed proteins, information was extracted from literature and genecards web portal (https://www.genecards.org/Guide/GeneCard\#compartments). The confidence score at genecards webportal was incorporated from literature, high throughput screen and similar text mining analyses. The 'unified confidence score' ranged from absence of localization evidence (value $=0$ ) and range from low confidence (value $=1)$ to high confidence (value $=5$ ).

Identification of differentially expressed mRNAs. The differentially expressed genes (DEGs) were analyzed using DEseq2 package in R (http://bioconductor.org/packages/ release/bioc/html/DESeq2.html). The results were analyzed and interpreted in R using 'plotPCA' function for Principal component analysis and volcano plot was generated using 'enhancedvolcano' package (http://bioconductor.org/ packages/ EnhancedVolcano.html).

GSEA (Gene Set Enrichment Analysis) and network analysis. To explore the pathways associated changes in patients with high-risk score, GSEA software was downloaded from the Broad Institute ( http://www. broadinstitute.org/gsea). For this analysis, patients with highest expression of ECM signature (top 50) were compared with patients with lowest expression (bottom 50). All genes were pre-ranked based on DESeq2-calculated $\log 2$ fold changes using R and target gene sets were "H: hallmark gene sets" and "C5.GO.MF.v7.2" with the number of permutations set at 1000. Enrichment statistic was set to 'weighted' and 'Signal2Noise' metric was used for ranking genes. $\mathrm{H}$ (hallmark gene sets, 50 gene sets), and C5 (ontology gene sets, 14,765 gene sets).

Gene interaction network analysis. Network analysis was performed to identify the interactions between the three genes with highest log-fold change compared to tumor using g:profiler ${ }^{65}$. GeneMANIA was used to analyze the string network analysis (http://genemania.org/) ${ }^{66}$.

Immune cell infiltration analysis. In this study, the immune cell infiltration landscape of high-and low- risk patients were obtained via CIBERSORT-ABS algorithm which quantifies absolute proportion of cells. Briefly, it is determined by calculating the median expression of all the genes in signature and is divided by gene expression level in whole sample ${ }^{67}$. The absolute scores calculated using this method can be used for both interand intra-sample comparisons.

Clustering analysis. Hierarchal clustering of correlation between immune cells and risk groups was generated using ward's methods. Hierarchal clusters and constellation plots were prepared using JMP-Pro (version 14.0.0, SAS Institute, Cary, USA).

Kaplan-Meier survival analysis. The Log-rank test was used to compare the survival distribution using Kaplan-Meier analysis using JMP-Pro (version 14.0.0, SAS Institute, Cary, USA).

Statistical analysis. The statistical significance of OS (Overall survival), DFS (Disease -free survival), PFS (Progression free-survival) and DSS (Disease-specific survival) was computed using Log-rank t-test through cBioportal. For Pearson's chi-square $(\chi 2)$ test, the numeric values of the risk score were split at the median and divided in 2 groups for comparison of patients in high and low-risk groups. GEPIA web-portal utilizes one-way ANOVA for calculation of differential expression between tumor and normal. GeneMANIA utilizes Geno-Ontology based weighing method that assigns score based on biological processes behind the interacting genes. For Gene Set enrichment analysis, the normalized gene enrichment score was kept at greater than $>2$ was considered strong. $p$ values of $<0.05$ were considered statistically significant.

\section{Data availability}

The study utilized The Cancer Genome Atlas (TCGA) Program and Gene Expression Omnibus (GEO) repositories, which are freely available to the public.

Received: 22 December 2020; Accepted: 22 March 2021

Published online: 07 April 2021

\section{References}

1. Siegel, R. L., Miller, K. D. \& Jemal, A. Cancer statistics, 2018. CA Cancer J. Clin. 68, 7-30. https://doi.org/10.3322/caac.21442 (2018).

2. Joosten, S. C. et al. Prognostic DNA methylation markers for renal cell carcinoma: a systematic review. Epigenomics 9, 1243-1257. https://doi.org/10.2217/epi-2017-0040 (2017).

3. Wei, J. H. et al. A CpG-methylation-based assay to predict survival in clear cell renal cell carcinoma. Nat. Commun. 6, 8699. https:// doi.org/10.1038/ncomms9699 (2015). 
4. Beksac, A. T. et al. Heterogeneity in renal cell carcinoma. Urol. Oncol. 35, 507-515. https://doi.org/10.1016/j.urolonc.2017.05.006 (2017).

5. Geiger, B. \& Yamada, K. M. Molecular architecture and function of matrix adhesions. Cold Spring Harb. Perspect. Biol. https://doi. org/10.1101/cshperspect.a005033 (2011).

6. Pickup, M. W., Mouw, J. K. \& Weaver, V. M. The extracellular matrix modulates the hallmarks of cancer. EMBO Rep. 15, 1243-1253. https://doi.org/10.15252/embr.201439246 (2014).

7. Spill, F., Reynolds, D. S., Kamm, R. D. \& Zaman, M. H. Impact of the physical microenvironment on tumor progression and metastasis. Curr. Opin. Biotechnol. 40, 41-48. https://doi.org/10.1016/j.copbio.2016.02.007 (2016).

8. Mushtaq, M. U. et al. Tumor matrix remodeling and novel immunotherapies: the promise of matrix-derived immune biomarkers. J. Immunother. Cancer 6, 65. https://doi.org/10.1186/s40425-018-0376-0 (2018).

9. Jiang, D. \& Lim, S. Y. Influence of immune myeloid cells on the extracellular matrix during cancer metastasis. Cancer Microenviron. 9, 45-61. https://doi.org/10.1007/s12307-016-0181-6 (2016).

10. Gao, Z. et al. A five-gene signature predicts overall survival of patients with papillary renal cell carcinoma. PLoS ONE 14, e0211491. https://doi.org/10.1371/journal.pone.0211491 (2019).

11. Ahluwalia, P., Kolhe, R. \& Gahlay, G. K. The clinical relevance of gene expression based prognostic signatures in colorectal cancer. Biochim. Biophys. Acta Rev. Cancer 1875, 188513. https://doi.org/10.1016/j.bbcan.2021.188513 (2021).

12. Supplitt, S., Karpinski, P., Sasiadek, M. \& Laczmanska, I. Current achievements and applications of transcriptomics in personalized cancer medicine. Int. J. Mol. Sci. https://doi.org/10.3390/ijms22031422 (2021).

13. Waz, S. et al. Structural and kinetic studies of the human nudix hydrolase MTH1 reveal the mechanism for its broad substrate specificity. J. Biol. Chem. 292, 2785-2794. https://doi.org/10.1074/jbc.M116.749713 (2017).

14. Ou, Q. et al. Nudix hydrolase 1 is a prognostic biomarker in hepatocellular carcinoma. Aging (Albany NY) 12, 7363-7379. https:// doi.org/10.18632/aging.103083 (2020).

15. Singh, R. \& Choi, B. K. Siglec1-expressing subcapsular sinus macrophages provide soil for melanoma lymph node metastasis. Elife https://doi.org/10.7554/eLife.48916 (2019).

16. Cassetta, L. et al. Human tumor-associated macrophage and monocyte transcriptional landscapes reveal cancer-specific reprogramming, biomarkers, and therapeutic targets. Cancer Cell 35, 588-602. https://doi.org/10.1016/j.ccell.2019.02.009 (2019).

17. Potere, N., Del Buono, M. G., Mauro, A. G., Abbate, A. \& Toldo, S. Low density lipoprotein receptor-related protein-1 in cardiac inflammation and infarct healing. Front Cardiovasc. Med. 6, 51. https://doi.org/10.3389/fcrm.2019.00051 (2019).

18. Xing, P. et al. Roles of low-density lipoprotein receptor-related protein 1 in tumors. Chin. J. Cancer 35, 6. https://doi.org/10.1186/ s40880-015-0064-0 (2016).

19. Zhan, P., Lv, X. J., Ji, Y. N., Xie, H. \& Yu, L. K. Increased lysyl oxidase-like 2 associates with a poor prognosis in non-small cell lung cancer. Clin. Respir. J. 12, 712-720. https://doi.org/10.1111/crj.12584 (2018).

20. Li, S. et al. Plasminogen activator inhibitor-1 in cancer research. Biomed. Pharmacother. 105, 83-94. https://doi.org/10.1016/j. biopha.2018.05.119 (2018).

21. Tsai, C. K. et al. Overexpression of PLOD3 promotes tumor progression and poor prognosis in gliomas. Oncotarget 9, 15705-15720. https://doi.org/10.18632/oncotarget.24594 (2018).

22. Li, S. S., Lian, Y. F., Huang, Y. L., Huang, Y. H. \& Xiao, J. Overexpressing PLOD family genes predict poor prognosis in gastric cancer. J. Cancer 11, 121-131. https://doi.org/10.7150/jca.35763 (2020).

23. Robinson, J. L., Feizi, A., Uhlen, M. \& Nielsen, J. A systematic investigation of the malignant functions and diagnostic potential of the cancer secretome. Cell Rep. 26, 2622-2635. https://doi.org/10.1016/j.celrep.2019.02.025 (2019).

24. Liu-Chittenden, Y. et al. RARRES2 functions as a tumor suppressor by promoting beta-catenin phosphorylation/degradation and inhibiting p38 phosphorylation in adrenocortical carcinoma. Oncogene 36, 3541-3552. https://doi.org/10.1038/onc.2016.497 (2017).

25. Cho, S. Y. et al. Amplification of transglutaminase 2 enhances tumor-promoting inflammation in gastric cancers. Exp. Mol. Med. 52, 854-864. https://doi.org/10.1038/s12276-020-0444-7 (2020).

26. Yuan, L. et al. Overexpression of COL3A1 confers a poor prognosis in human bladder cancer identified by co-expression analysis. Oncotarget 8, 70508-70520. https://doi.org/10.18632/oncotarget.19733 (2017).

27. Li, L., Zhang, R., Liu, Y. \& Zhang, G. ANXA4 activates JAK-STAT3 signaling by interacting with ANXA1 in basal-like breast cancer. DNA Cell Biol. 39, 1649-1656. https://doi.org/10.1089/dna.2020.5570 (2020).

28. Wei, B., Guo, C., Liu, S. \& Sun, M. Z. Annexin A4 and cancer. Clin. Chim. Acta 447, 72-78. https://doi.org/10.1016/j.cca.2015.05. 016 (2015).

29. Gonzalez-Gonzalez, L. \& Alonso, J. Periostin: a matricellular protein with multiple functions in cancer development and progression. Front Oncol. 8, 225. https://doi.org/10.3389/fonc.2018.00225 (2018).

30. Lockhart, M., Wirrig, E., Phelps, A. \& Wessels, A. Extracellular matrix and heart development. Birth Defects Res. A Clin. Mol. Teratol. 91, 535-550. https://doi.org/10.1002/bdra.20810 (2011).

31. Walker, C., Mojares, E. \& Del Rio Hernandez, A. Role of extracellular matrix in development and cancer progression. Int. J. Mol. Sci. https://doi.org/10.3390/ijms19103028 (2018).

32. Kim, S. H., Turnbull, J. \& Guimond, S. Extracellular matrix and cell signalling: the dynamic cooperation of integrin, proteoglycan and growth factor receptor. J. Endocrinol. 209, 139-151. https://doi.org/10.1530/JOE-10-0377 (2011).

33. de Visser, K. E., Eichten, A. \& Coussens, L. M. Paradoxical roles of the immune system during cancer development. Nat. Rev. Cancer 6, 24-37. https://doi.org/10.1038/nrc1782 (2006).

34. Grolman, J. M., Weinand, P. \& Mooney, D. J. Extracellular matrix plasticity as a driver of cell spreading. Proc. Natl. Acad. Sci. USA 117, 25999-26007. https://doi.org/10.1073/pnas.2008801117 (2020).

35. Poltavets, V., Kochetkova, M., Pitson, S. M. \& Samuel, M. S. The role of the extracellular matrix and its molecular and cellular regulators in cancer cell plasticity. Front Oncol. 8, 431. https://doi.org/10.3389/fonc.2018.00431 (2018).

36. Bougherara, H. et al. Real-time imaging of resident $\mathrm{T}$ cells in human lung and ovarian carcinomas reveals how different tumor microenvironments control T lymphocyte migration. Front Immunol. 6, 500. https://doi.org/10.3389/fimmu.2015.00500 (2015).

37. Henke, E., Nandigama, R. \& Ergun, S. Extracellular matrix in the tumor microenvironment and its impact on cancer therapy. Front Mol. Biosci. 6, 160. https://doi.org/10.3389/fmolb.2019.00160 (2019).

38. Nallanthighal, S., Heiserman, J. P. \& Cheon, D. J. The role of the extracellular matrix in cancer stemness. Front Cell Dev. Biol. 7, 86. https://doi.org/10.3389/fcell.2019.00086 (2019).

39. Schaaf, M. B., Garg, A. D. \& Agostinis, P. Defining the role of the tumor vasculature in antitumor immunity and immunotherapy. Cell Death Dis. 9, 115. https://doi.org/10.1038/s41419-017-0061-0 (2018).

40. Vella, D., Zoppis, I., Mauri, G., Mauri, P. \& Di Silvestre, D. From protein-protein interactions to protein co-expression networks: a new perspective to evaluate large-scale proteomic data. EURASIP J. Bioinf. Syst. Biol. 2017, 6. https://doi.org/10.1186/s13637017-0059-z (2017).

41. Bergamaschi, A. et al. Extracellular matrix signature identifies breast cancer subgroups with different clinical outcome. J. Pathol. 214, 357-367. https://doi.org/10.1002/path.2278 (2008).

42. Jiang, K., Liu, H., Xie, D. \& Xiao, Q. Differentially expressed genes ASPN, COL1A1, FN1, VCAN and MUC5AC are potential prognostic biomarkers for gastric cancer. Oncol. Lett. 17, 3191-3202. https://doi.org/10.3892/ol.2019.9952 (2019). 
43. Chen, D., Chen, D., Cao, D., Hu, J. \& Yao, Y. A signature based on survival-related genes identifies high-risk glioblastomas harboring immunosuppressive and aggressive ECM characteristics. Zhong Nan Da Xue Xue Bao Yi Xue Ban 43, 368-382. https://doi.org/ 10.11817/j.issn.1672-7347.2018.04.006 (2018).

44. Majo, S., Courtois, S., Souleyreau, W., Bikfalvi, A. \& Auguste, P. Impact of extracellular matrix components to renal cell carcinoma behavior. Front Oncol. 10, 625. https://doi.org/10.3389/fonc.2020.00625 (2020).

45. Boguslawska, J. et al. Expression of genes involved in cellular adhesion and extracellular matrix remodeling correlates with poor survival of patients with renal cancer. J. Urol. 195, 1892-1902. https://doi.org/10.1016/j.juro.2015.11.050 (2016).

46. Stadler, K., Goldberg, I. J. \& Susztak, K. The evolving understanding of the contribution of lipid metabolism to diabetic kidney disease. Curr. Diab. Rep. 15, 40. https://doi.org/10.1007/s11892-015-0611-8 (2015).

47. Lowrance, W. T., Ordonez, J., Udaltsova, N., Russo, P. \& Go, A. S. CKD and the risk of incident cancer. J. Am. Soc. Nephrol. 25, 2327-2334. https://doi.org/10.1681/ASN.2013060604 (2014).

48. Hu, S. L. et al. The nephrologist's tumor: basic biology and management of renal cell carcinoma. J. Am. Soc. Nephrol. 27, 2227-2237. https://doi.org/10.1681/ASN.2015121335 (2016).

49. Paluskievicz, C. M. et al. T regulatory cells and priming the suppressive tumor microenvironment. Front Immunol. 10, 2453. https:// doi.org/10.3389/fimmu.2019.02453 (2019).

50. Davidsson, S. et al. Infiltration of M2 macrophages and regulatory T cells plays a role in recurrence of renal cell carcinoma. Eur. Urol. Open Sci. 20, 62-71. https://doi.org/10.1016/j.euros.2020.06.003 (2020).

51. Motzer, R. J. et al. Nivolumab plus ipilimumab versus sunitinib in first-line treatment for advanced renal cell carcinoma: extended follow-up of efficacy and safety results from a randomised, controlled, phase 3 trial. Lancet Oncol. 20, 1370-1385. https://doi.org/ 10.1016/S1470-2045(19)30413-9 (2019).

52. Mousavi-Niri, N., Naseroleslami, M. \& Hadjati, J. Anti-regulatory T cell vaccines in immunotherapy: focusing on FoxP3 as target. Hum. Vaccin. Immunother. 15, 620-624. https://doi.org/10.1080/21645515.2018.1545625 (2019).

53. Bagati, A. et al. Integrin alphavbeta6-TGFbeta-SOX4 pathway drives immune evasion in triple-negative breast cancer. Cancer Cell 39, 54-67. https://doi.org/10.1016/j.ccell.2020.12.001 (2021).

54. Rossow, L. et al. LOX-catalyzed collagen stabilization is a proximal cause for intrinsic resistance to chemotherapy. Oncogene 37, 4921-4940. https://doi.org/10.1038/s41388-018-0320-2 (2018).

55. Salmon, H. \& Donnadieu, E. Within tumors, interactions between $\mathrm{T}$ cells and tumor cells are impeded by the extracellular matrix. Oncoimmunology 1, 992-994. https://doi.org/10.4161/onci.20239 (2012).

56. Wang, X. et al. Hybrid $\mathrm{pH}$-sensitive nanogels surface-functionalized with collagenase for enhanced tumor penetration. J. Colloid Interface Sci. 525, 269-281. https://doi.org/10.1016/j.jcis.2018.04.084 (2018).

57. Carbon, S. et al. AmiGO: online access to ontology and annotation data. Bioinformatics 25, 288-289. https://doi.org/10.1093/bioin formatics/btn615 (2009).

58. Gao, J. et al. Integrative analysis of complex cancer genomics and clinical profiles using the cBioPortal. Sci. Signal https://doi.org/ 10.1126/scisignal.2004088 (2013).

59. Tang, Z., Kang, B., Li, C., Chen, T. \& Zhang, Z. GEPIA2: an enhanced web server for large-scale expression profiling and interactive analysis. Nucleic Acids Res. 47, W556-W560. https://doi.org/10.1093/nar/gkz430 (2019).

60. Aguirre-Gamboa, R. et al. SurvExpress: an online biomarker validation tool and database for cancer gene expression data using survival analysis. PLOS ONE 8, e74250. https://doi.org/10.1371/journal.pone.0074250 (2013).

61. Jan, Y. H., Lai, T. C., Yang, C. J., Huang, M. S. \& Hsiao, M. A co-expressed gene status of adenylate kinase 1/4 reveals prognostic gene signature associated with prognosis and sensitivity to EGFR targeted therapy in lung adenocarcinoma. Sci. Rep. 9, 12329. https://doi.org/10.1038/s41598-019-48243-9 (2019).

62. Zuo, S., Zhang, X. \& Wang, L. A RNA sequencing-based six-gene signature for survival prediction in patients with glioblastoma. Sci. Rep. 9, 2615. https://doi.org/10.1038/s41598-019-39273-4 (2019).

63. Ahluwalia, P. et al. Identification and clinical validation of a novel 4 gene-signature with prognostic utility in colorectal cancer. Int. J. Mol. Sci. https://doi.org/10.3390/ijms20153818 (2019).

64. Ahluwalia, P. et al. Immunogenomic gene signature of cell-death associated genes with prognostic implications in lung cancer. Cancers (Basel) https://doi.org/10.3390/cancers13010155 (2021).

65. Raudvere, U. et al. g:Profiler: a web server for functional enrichment analysis and conversions of gene lists (2019 update). Nucleic Acids Res. 47, W191-W198. https://doi.org/10.1093/nar/gkz369 (2019).

66. Warde-Farley, D. et al. The GeneMANIA prediction server: biological network integration for gene prioritization and predicting gene function. Nucleic Acids Res. 38, W214-220. https://doi.org/10.1093/nar/gkq537 (2010).

67. Chen, B., Khodadoust, M. S., Liu, C. L., Newman, A. M. \& Alizadeh, A. A. Profiling tumor infiltrating immune cells with CIBERSORT. Methods Mol. Biol. 1711, 243-259. https://doi.org/10.1007/978-1-4939-7493-1_12 (2018).

\section{Author contributions}

P.A. and R.K. conceived, designed, and wrote the manuscript. P.A. and M.A. performed the analysis and interpreted the data. A.M., N.S., V.K., M.R., A.R. helped with the manuscript and data review. R.K. supervised the study. All authors read and approved the final manuscript.

\section{Funding}

This study was funded by the start-up grant awarded to RK by Medical College of Georgia by Augusta University.

\section{Competing interests}

The authors declare no competing interests.

\section{Additional information}

Supplementary Information The online version contains supplementary material available at https://doi.org/ 10.1038/s41598-021-86888-7.

Correspondence and requests for materials should be addressed to R.K.

Reprints and permissions information is available at www.nature.com/reprints.

Publisher's note Springer Nature remains neutral with regard to jurisdictional claims in published maps and institutional affiliations. 
(c) (i) Open Access This article is licensed under a Creative Commons Attribution 4.0 International cc) License, which permits use, sharing, adaptation, distribution and reproduction in any medium or format, as long as you give appropriate credit to the original author(s) and the source, provide a link to the Creative Commons licence, and indicate if changes were made. The images or other third party material in this article are included in the article's Creative Commons licence, unless indicated otherwise in a credit line to the material. If material is not included in the article's Creative Commons licence and your intended use is not permitted by statutory regulation or exceeds the permitted use, you will need to obtain permission directly from the copyright holder. To view a copy of this licence, visit http://creativecommons.org/licenses/by/4.0/.

(C) The Author(s) 2021 